Supplement of Ann. Geophys., 38, 1101-1113, 2020

https://doi.org/10.5194/angeo-38-1101-2020-supplement

(C) Author(s) 2020. This work is distributed under

the Creative Commons Attribution 4.0 License.

(c) (1)

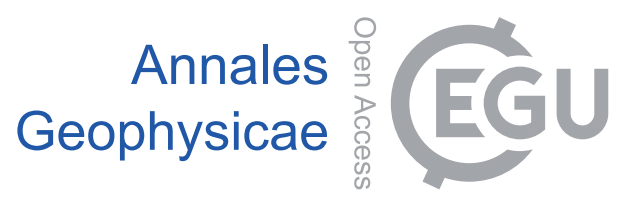

Supplement of

\title{
Ducting of incoherent scatter radar waves by field-aligned irregularities
}

Michael T. Rietveld and Andrew Senior

Correspondence to: Michael T. Rietveld (mike.rietveld@ eiscat.uit.no)

The copyright of individual parts of the supplement might differ from the CC BY 4.0 License. 
This supplement shows the results of the search for WAILES as described in Section 2 of the paper "Ducting of incoherent scatter radar waves by field-aligned irregularities"

by M. T. Rietveld and A. Senior

This supplement should be useful for further studies of the WAILE phenomena using EISCAT UHF radar data.

We highlight here two dates where WAILEs were observed at the unusually low $\mathrm{HF}$ frequency of $4.544 \mathrm{MHz}$ for this phenomenon:

2013-02-18 15:30-17:0९

$2012-02-12$

a1_heating_all_annotated.log

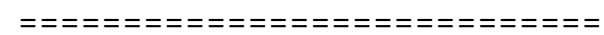

\# Line format:

\# YYYY-mm-dd HH min_f_heat max_foF2 status1 status0 statusX notes

\# status1 is:

\# X Experiment unsuitable

\# Q Radar quick-look plot unavailable/unsuitable

\# Y WAILEs observed

\# N No WAILEs observed

\# U Unclear

\#

\# status0 and statusX are (for 0 - and X-mode respectively):

\# Y WAILEs observed

\# N No WAILEs observed

\# U Unclear

\# - polarisation not used

\# anything else is meaningless

\#

\# max_foF2 $=$ NaN indicates no data in the hour

$\#$

2001-02-16 16 6.2000 NaN Q Q Q

2001-02-17 16 5.4230 NaN Q Q Q

2001-02-2017 6.2000 7.2 Q Q Q

2001-11-08 156.20009 .1 U U -

2001-11-08 $16 \quad 6.20007 .9$ U U -

2001-11-08 $17 \quad 5.42307 .0 \mathrm{U}$ U -

2001-11-08 185.42305 .4 U U -

2001-11-09 $157.95308 .8 \mathrm{U}$ U -

2001-11-09 165.42307 .2 U U -

2001-11-09 175.42306 .4 U U -

2001-11-10 156.77008 .0 U U -

2001-11-10 $16 \quad 6.7700 \quad 7.3$ U U -

2001-11-10 $17 \quad 5.42306 .3 \cup$ U -

2001-11-11 $155.42308 .7 \mathrm{Y} Y-$ 2001-11-11 $165.42307 .8 \mathrm{Y} Y-$ 2001-11-11 $17 \quad 5.42306 .6$ Y Y -

2001-11-12 155.42308 .9 U U -

2001-11-12 $165.42308 .4 \mathrm{U}$ U -

2001-11-12 $175.42307 .5 \mathrm{U}$ U - heater scanning

2001-11-12 $185.42306 .0 \mathrm{U}$ U -

2001-11-17 10 5.4230 10.6 X X X D-region

2001-11-17 11 5.4230 11.6 X X X

2001-11-17 12 5.4230 NaN X X X 
2002-03-05 $175.42305 .8 \mathrm{U}$ U - disturbed conditions 2002-03-05 20 5.42305.7 U U -

2002-03-06 175.42305 .3 U U - disturbed conditions 2002-03-06 $185.42304 .9 \cup U$ 2002-03-06 $195.42305 .9 \cup U$ -

2002-03-07 175.42309 .4 U U 2002-03-07 $185.28309 .4 \quad U$ U 2002-๑3-๑7 $195.28305 .8 \cup U$ -

2002-03-๑8 $17 \quad 5.42308 .7 \cup U$ 2002-03-08 186.9600 8.8 Y Y 2002-03-08 $196.55005 .8 \mathrm{U}$ U 2002-03-08 $205.42305 .7 \quad \mathrm{U} U$ 2002-03-๑8 $215.30305 .1 Y Y$ -

2002-03-09 175.42305 .8 U U - disturbed conditions 2002-03-09 $185.42305 .8 \mathrm{U} U$ 2002-03-09 $195.42305 .8 \mathrm{U} U$ 2002-03-09 20 $5.42304 .7 \cup U$ 2002-03-09 215.42305.2 U U -

2002-03-10 $18 \quad 5.4230 \quad 7.2$ Y $Y$ 2002-03-10 195.42306 .3 Y $Y$ -

2002-07-22 09 5.4230 6.3 X X X PMSE

2002-07-22 105.42306.4 X X X 2002-07-22 $115.42306 .7 \times \times \times$ 2002-07-22 $125.42306 .7 \times \times \times$ 2002-07-22 135.42306.1 X X X 2002-07-22 $145.42305 .3 \times \times X$ 2002-07-22 15 5.4230 5.9 X X X 2002-07-22 $215.42300 .0 \times \times X$ 2002-07-22 $225.42300 .0 \times \times X$ 2002-07-22 $237.95300 .0 \times \times X$ 2002-07-24 $005.42300 .0 \times \times X$ 2002-07-24 $065.42306 .1 \times \times X$ 2002-07-24 07 5.4230 6.1 X X X 2002-07-24 08 5.42306.0 X X X 2002-07-24 $095.42305 .9 \times \times X$ 2002-07-24 105.42306.0 X X X 2002-07-24 115.42306.1 X X X 2002-07-24 12 5.42306.0 X X X 2002-07-24 145.42305.8 X X X 2002-07-24 22 5.42305.6 X X X 2002-07-25 $105.42306 .1 \times \times \times$ 2002-07-25 $115.42306 .2 \times \times \times$ 2002-07-25 $125.42306 .0 \times \times \times$ 2002-07-25 $135.42306 .0 \times \times X$ 2002-08-01 125.42305 .6 X X X PMSE 2002-08-01 $135.42305 .5 \times \times \times$ 2002-08-02 $125.42305 .1 \times \times \times$ 2002-09-25 117.95009.5 X X X tune

2002-09-25 $127.95008 .9 U U$ - radar scanning 2002-09-25 $136.20009 .3 \mathrm{U} U$ 2002-09-25 $146.20009 .5 \mathrm{U} U$ 2002-09-25 $167.10009 .3 \cup U$ 2002-09-25 $177.10009 .0 \cup U$ -

2002-09-26 $127.10009 .8 \mathrm{U} U$ - radar scanning 2002-09-26 $137.100010 .0 \quad U$ U 2002-09-26 $147.100010 .0 \quad$ U U 2002-09-26 15 7.1000 10.6 Q Q Q 
2002-09-28 $127.950010 .3 \mathrm{U}$ U - radar scanning

2002-09-28 $137.950010 .3 \mathrm{U}$ U -

2002-09-28 $17 \quad 7.95008 .0$ U U -

2002-11-08 15 5.3030 NaN U U 2002-11-08 16 5.3740 NaN U U -

2003-03-06 $\odot 85.42306 .5 \times \times X$ D-region

2003-03-06 $095.42307 .3 \times \times \times$

2003-03-06 $105.42307 .7 \times \times \times$

2003-03-06 $116.77007 .7 \times \times \times$

2003-03-06 $125.42307 .4 \times \times \times$

2003-03-06 $136.20007 .5 \times \times x$

2003-03-06 $14 \quad 6.20005 .4 \times \times \times$

2003-03-06 $156.20007 .2 \times \times x$

2003-06-20 12 5.4230 6.2 X X X short pulses

2003-06-27 08 5.4230 4.9 X X X PMSE?

2003-06-27 $095.42305 .5 \times \times X$

2003-06-30 07 5.4230 NaN X X X PMSE?

2003-06-30 $\odot 8$ 5.4230 NaN X X X

2003-06-30 $\odot 95.42305 .2 \times \times \times$

2003-06-3010 5.4230 5.4 X X X

2003-07-01 05 5.4230 4.9 X X X PMSE

2003-07-01 $065.42305 .5 \times \times X$

2003-07-01 $075.42305 .3 \times \times x$

2003-07-01 08 5.4230 5.5 X X X

2003-07-01 $\odot 95.42305 .5 \times \times \times$

2003-07-01 $105.42305 .6 \times \times x$

2003-07-01 $115.42305 .7 \times \times X$

2003-07-02 07 5.42305.8 X X X PMSE

2003-07-02 08 5.42306.2 X XX

2003-07-02 $095.42306 .3 \times \times x$

2003-07-02 $105.42306 .5 \times \times X$

2003-08-01 $105.42304 .9 \times \times \times$ XMSE?

2003-08-04 $075.42305 .8 \times X \times$ XMSE?

2003-08-04 08 5.42306.4 X X X

2003-08-04 $095.42306 .0 \times \times x$

2003-08-04 $105.42306 .1 \times \times x$

2003-08-04 $115.42306 .3 \times \times \times$

2003-11-06 $095.42306 .6 \times \times \times$ short pulses (LT)

2003-11-06 $116.20007 .9 \times \times \times$

2003-11-06 $126.20006 .9 \times \times \times$

2003-11-20 20 5.4230 7.7 U U - disturbed conditions 2003-11-20 215.423010 .1 U U -

2003-11-22 19 5.4230 7.8 U U - disturbed conditions 2003-11-22 205.42307 .9 U U -

2003-11-24 $145.42305 .3 \times \times \times$ D-region

2003-11-24 21 5.42306.5 X X X $3 \mathrm{~Hz}$

2003-11-25 14 5.4230 5.2 X X X D-region

2003-11-27 $145.42306 .4 \times \times \times$ no UHF

2003-11-27 15 5.4230 5.5 X X X

2004-02-26 $145.42306 .2 \times \times \times$ no UHF

2004-05-12 $125.42305 .4 \times \times \times$ test

2004-06-๑9 125.42305 .4 U U - radar scanning

2004-06-10 21 5.4230 0.0 X X X MARE

2004-06-12 $135.42306 .1 \times \times \times$ short pulses

2004-06-12 $155.42306 .0 \times \times x$ 
2004-06-12 $175.42305 .7 \times \times \times$ unsuitable UHF data

2004-06-12 18 5.4230 5.5 XXX

2004-06-30 10 5.4230 5.8 X X X PMSE

2004-06-3011 5.42305.4 X XX

2004-06-3012 5.4230 5.5 XXX

2004-06-30 13 5.4230 5.4 X XX

2004-07-02 $095.42305 .2 \times \times X$ PMSE

2004-07-02 $105.42305 .3 \times \times X$

2004-07-02 $115.42305 .3 \times \times x$ 2004-07-02 $125.42305 .3 \times \times X$ 2004-07-05 $075.42305 .7 \times X \times$ PMSE 2004-07-05 $\odot 8$ 5.4230 NaN XXX 2004-07-05 095.4230 NaN $X X X$ 2004-07-05 $105.4230 \mathrm{NaN} X X X$ 2004-07-06 $\odot 85.42305 .7 \times X \times$ PMSE 2004-07-06 09 5.4230 NaN XXX 2004-07-06 $105.42305 .4 \times \times x$ 2004-07-06 $115.42305 .6 \times \times X$ 2004-07-07 $075.42305 .6 \times \times X$ PMSE 2004-07-07 $085.42305 .5 \times \times X$ 2004-07-07 $095.42305 .5 \times \times x$ 2004-07-07 10 5.42306.7 X X X 2004-07-07 11 5.4230 5.7 XXX 2004-07-08 $075.42305 .6 \times \times \times$ X PMSE 2004-07-08 $\odot 8$ 5.4230 NaN $X X X$ 2004-07-08 $\odot 9$ 5.4230 NaN $X X X$ 2004-07-08 $105.4230 \mathrm{NaN} X X X$ 2004-07-10 $\odot 75.42305 .4 \times \times X$ XMSE 2004-07-10 08 5.4230 5.7 X X X 2004-07-10 $095.42306 .4 \times \times x$ 2004-07-10 10 5.42306.0 X X X 2004-07-10 11 5.4230 5.5 X X X 2004-07-11 07 5.4230 5.4 X X X PMSE 2004-07-11 $\odot 85.42305 .7 \times \times X$ 2004-07-11 $095.42306 .0 \times \times x$ 2004-07-11 $105.42305 .8 \times \times X$ 2004-07-12 $\odot 7$ 5.4230 5.0 X X X PMSE 2004-07-12 08 5.4230 4.8 X X X 2004-07-12 $095.42304 .9 \times \times X$ 2004-07-12 10 5.4230 5.1 X X X 2004-07-12 $115.42305 .3 \times \times x$ 2004-07-13 $075.42305 .1 \times \times \times$ PMSE 2004-07-13 08 5.4230 5.0 X X X 2004-07-14 $07 \quad 6.96006 .5 \times \times \times$ XMSE 2004-07-14 $085.42306 .4 \times \times X$ 2004-07-14 $095.42306 .4 \times \times X$ 2004-07-14 10 5.4230 6.3 X X X 2004-07-15 $075.42305 .6 \times \times \times$ PMSE 2004-07-15 $085.42306 .2 \times \times X$ 2004-07-15 $095.42306 .7 \times \times x$ 2004-07-15 $105.42306 .0 \times \times \times$ 2004-07-15 $115.42305 .6 \times \times x$ 2004-10-01 $146.20007 .2 \times \times \times$ short pulses 2004-10-01 15 5.4230 7.1 X X X unsuitable UHF data 2004-10-01 $165.42307 .1 \times \times x$ 2004-10-02 $116.20007 .5 \times \times \times$ short pulses 2004-10-02 $126.20007 .4 \times \times X$ 2004-10-02 $136.20007 .5 \times \times x$ 2004-10-05 $105.42305 .5 \times \times \times$ short pulses/unsuitable UHF data 2004-10-05 $115.42305 .8 \times \times x$ 2004-10-05 $125.42305 .9 \times \times x$ 2004-10-07 $115.42309 .2 \times \times \times$ unsuitable UHF data 2004-10-07 $125.42307 .7 \times \times \times$ no UHF 2004-10-07 13 5.42306.7 X X X 
2004-10-07 $165.423010 .3 \times \times \times$

2004-10-08 $115.42305 .9 \times \times \times$ unsuitable UHF data

2004-10-08 $125.42306 .5 \times \times \times$

2004-10-08 $135.42306 .3 \times \times x$

2005-06-01 08 5.4230 $0.0 \times \times \times$ no UHF

2005-06-01 $095.42300 .0 \times \times \times$

2005-06-01 $105.42300 .0 \times \times \times$

2005-06-01 $115.42300 .0 \times \times \times$

2005-06-01 $125.42300 .0 \times \times \times$

2005-06-01 $13 \quad 5.42300 .0 \times \times \times$

2005-06-29 08 5.4230 4.9 X X X

2005-06-29 $095.42305 .3 \times \times X$

2005-07-04 07 5.4230 5.5 X X X PMSE

2005-07-04 08 5.4230 5.5 X X X

2005-07-04 $\odot 95.42305 .7 \times \times x$

2005-07-04 $105.42305 .7 \times \times x$ 2005-07-04 $115.42305 .4 \times \times x$ 2005-07-04 $125.42305 .3 \times \times x$ 2005-07-05 $07 \quad 5.42306 .1 \times \times X$ 2005-07-05 08 5.42306.0 XXX 2005-07-05 09 5.4230 NaN $X X X$ 2005-07-05 10 5.4230 NaN $X X X$ 2005-07-05 $115.4230 \mathrm{NaN} X \times X$ 2005-07-05 $125.42305 .5 \times \times X$ 2005-07-06 $07 \quad 5.42306 .6 \times \times x$ 2005-07-06 08 5.4230 6.0 XXX 2005-07-06 $\odot 95.42306 .0 \times \times X$ 2005-07-06 $105.42305 .8 \times \times x$ 2005-07-07 $\odot 95.42305 .7 \times \times X$ 2005-07-07 $105.42305 .5 \times \times x$ 2005-07-07 $115.42305 .6 \times \times x$ 2005-07-07 $125.42305 .8 \times \times \times$ 2005-07-08 $07 \quad 5.42305 .0 \times \times \times$ 2005-07-08 08 5.4230 $5.7 \times \times x$ 2005-07-08 $\odot 95.42305 .7 \times \times x$ 2005-07-08 $105.42305 .4 \times \times \times$ 2005-07-08 $115.42305 .4 \times \times \times$ 2005-07-08 $125.42305 .4 \times \times x$ 2005-07-09 $075.42305 .8 \times \times \times$ 2005-07-09 08 5.42306.4 $6 \times \times$ 2005-07-09 $\odot 95.42307 .0 \times \times \times$ 2005-07-09 $105.42307 .5 \times \times \times$ 2005-07-09 $115.42307 .1 \times \times \times$ 2005-07-09 $125.42306 .8 \times \times x$ 2005-07-10 $07 \quad 5.42305 .1 \times \times \times$ 2005-10-04 $\odot 9$ 5.4230 NaN X X X D-region 2005-10-04 $105.4230 \mathrm{NaN} X X X$ 2005-10-04 $11 \quad 5.4230 \mathrm{NaN} \times \mathrm{X} X$ 2005-10-04 12 5.4230 NaN X X X 2005-10-05 $\odot 9$ 5.4230 NaN $X X X$ No UHF 2005-10-05 10 5.4230 NaN X X X 2005-10-05 11 5.4230 NaN X X X 2005-10-05 $12 \quad 5.4230$ NaN $X X X$ 2005-10-05 13 5.4230 NaN X X X 2005-10-05 14 5.4230 NaN X X X 2005-10-07 $\odot 8$ 5.4230 NaN $X X X$ No UHF 2005-10-07 $\odot 9$ 5.4230 NaN X X X 2005-10-07 10 5.4230 NaN X X X 2005-10-08 08 5.4230 NaN $X X X$ No UHF 2005-10-08 $\odot 9$ 5.4230 NaN X X X 2005-10-08 $105.4230 \mathrm{NaN} X X X$ 2005-10-08 11 5.4230 NaN $X X X$ 2005-10-08 16 5.4230 NaN X X X 2005-10-08 17 5.4230 NaN X X X 
2005-10-08 18 5.4230 NaN X X X

2005-10-10 08 5.4230 5.4 X X X No UHF

2005-10-10 $\odot 95.42305 .6 \times \times \times$

2005-10-10 $105.42305 .6 \times \times \times$

2005-10-10 $115.42306 .0 \times \times \times$

$2005-10-10125.42305 .7 \times \times \times$

2005-10-10 13 5.4230 5.4 X X X

2005-10-11 $115.42306 .1 \times \times \times$ unsuitable UHF data

2005-10-12 13 5.3400 5.9 X X X unsuitable UHF data

2005-10-12 $145.35006 .1 \times \times \times$

2005-10-12 $155.34005 .6 \times \times \times$

2005-10-13 $095.42306 .6 \times \times \times$ unsuitable UHF data

2005-10-13 $105.42306 .6 \times \times x$

2005-10-13 $115.42306 .9 \times \times \times$

2005-10-13 $126.20006 .3 \times \times \times$

2005-10-13 $136.20006 .3 \times \times x$

2005-12-07 11 5.42305.3 X X X test

2006-01-18 125.42305 .9 U U -

2006-01-21 13 5.42305.4 X X X tune

2006-01-21 14 5.4230 5.8 X X X no UHF

2006-07-28 16 5.4230 NaN X X X PMSE

2006-07-28 17 5.4230 NaN X X X

2006-07-28 18 5.4230 NaN X X X

2006-07-28 $195.4230 \mathrm{NaN} X X X$

2006-07-28 20 5.4230 NaN X X X

2006-07-28 21 5.4230 NaN X X X

2006-07-28 22 5.4230 NaN X X X

2006-07-28 23 5.4230 NaN X X X

2006-07-29 18 5.4230 NaN X X X PMSE

2006-07-29 19 5.4230 NaN X X X

2006-07-29 20 5.4230 NaN X X X

2006-07-29 21 5.4230 NaN X X X

2006-07-29 22 5.4230 NaN X X X

2006-07-29 23 5.4230 NaN X X X

2006-07-30 $\odot 05.42304 .9 \times \times X$

2006-08-03 $185.42305 .7 \times \times \times$ PMSE

2006-08-04 18 5.4230 4.9 X X X PMSE

2006-08-04 $195.42304 .7 \times \times X$

2006-08-04 20 5.4230 5.6 X X X

2006-08-04 $21 \quad 5.42305 .8 \times \times \times$

2006-08-04 22 5.42305.6 X X X

2006-08-04 23 5.4230 5.4 XXX

2006-08-12 08 5.4230 NaN $X X X$ PMSE

2006-08-12 $\odot 9$ 5.4230 NaN X X X

2006-08-12 10 5.4230 NaN X X X

2006-08-13 07 5.4230 NaN X X X PMSE

2006-08-13 $\odot 8$ 5.4230 NaN X X X

2006-08-13 $\odot 9$ 5.4230 NaN X X X

2006-08-13 $105.4230 \mathrm{NaN} X X X$

2006-10-24 $105.42306 .0 \times \times \times$ unsuitable UHF data

2006-10-24 $115.42306 .4 \times \times \times$

2006-10-24 $125.42305 .9 \times \times \times$

2006-11-2019 5.4230 $\odot .0 \times \times \times$ no UHF

2006-11-21 16 5.4230 NaN X X X no UHF

2006-11-21 17 5.4230 NaN X X X

2006-11-21 18 5.4230 NaN X X X

2006-11-24 08 5.4230 NaN $X X X$ no UHF

2006-11-24 $\odot 9$ 5.4230 NaN $X X X$

2006-11-27 10 5.4230 NaN X X X no UHF

2006-12-08 $105.42300 .0 \times \times \times$ PMWE

2006-12-08 $115.42300 .0 \times \times \times$

2006-12-08 $125.42300 .0 \times \times \times$

2007-02-23 $095.42305 .6 U$ U - radar scanning 
2007-06-28 $06 \quad 5.42304 .8 \times \times \times$ X PMSE

2007-06-28 $07 \quad 5.42304 .9 \times \times X$

2007-06-28 08 5.4230 5.2 X X X 2007-06-28 $\odot 95.42305 .1 \times \times x$ 2007-06-28 $105.42305 .0 \times \times \times$ 2007-06-28 $115.42305 .0 \times \times X$ 2007-07-10 $\odot 75.42305 .2 \times \times \times$ PMSE 2007-07-10 08 5.4230 5.6 X X X 2007-07-10 $095.42305 .1 \times \times x$ 2007-07-10 $105.42305 .0 \times \times \times$ 2007-07-12 13 5.4230 4.9 X X X PMSE 2007-07-13 $\odot 7$ 5.4230 5.0 X X X PMSE 2007-07-13 08 5.4230 4.7 X X X 2007-07-13 $\odot 95.42304 .7 \times \times x$ 2007-07-13 $115.42304 .7 \times \times x$ 2007-07-13 $12 \quad 5.42305 .7 \times x \times$ 2007-07-17 16 7.9530 8.6 X X X Test 2007-07-18 07 7.9530 7.3 X X X PMSE 2007-07-18 08 7.9530 10.7 X X X 2007-07-18 $097.95308 .1 \times \times x$ 2007-07-18 $105.42306 .5 \times \times x$ 2007-07-18 $115.42305 .8 \times \times \times$ 2007-07-18 $125.42306 .1 \times \times X$ 2007-07-19 07 5.4230 8.4 X X X PMSE 2007-07-19 08 5.4230 4.8 X X X 2007-07-19 $095.42304 .9 \times \times \times$ 2007-08-29 $125.42304 .8 \times \times \times$ Test 2007-10-10 09 5.4230 5.5 X X X D-region 2007-10-11 $\odot 9$ 5.4230 5.4 X X X D-region 2007-10-11 $105.42305 .5 \times \times \times$ 2007-10-11 $105.42305 .5 \times \times \times$ 2007-10-11 $115.42305 .4 \times \times x$ 2007-10-11 $125.42305 .2 \times \times \times$ 2007-10-13 $\odot 8$ 5.4230 5.5 X X X D-region 2007-10-13 $\odot 95.42305 .6 \times \times \times$ 2007-10-13 $105.42305 .7 \times \times \times$ 2007-10-13 $115.42305 .7 \times \times x$ 2007-10-13 $12 \quad 5.42305 .7 \times \times X$ 2007-10-14 $\odot 8$ 5.4230 5.4 X X X D-region 2007-10-14 $\odot 95.42305 .3 \times \times \times$ 2007-10-14 10 5.4230 5.8 X X X 2007-10-14 $115.42305 .7 \times \times \times$ 2007-10-14 $125.42305 .2 \times \times X$ 2007-10-15 $\odot 95.42305 .7 \times$ X X D-region 2007-10-15 $105.42305 .7 \times \times \times$ 2007-10-15 $115.42305 .7 \times \times \times$ 2007-10-16 $\odot 95.42305 .7 \times \times \times$ D-region 2007-10-16 $105.42305 .4 \times \times \times$ 2007-10-16 $115.42305 .4 \times \times X$ 2007-10-17 09 5.4230 5.0 X X X D-region 2007-10-17 $105.42305 .1 \times \times \times$ 2007-10-17 $115.42305 .6 \times \times \times$ 2007-10-18 $095.42306 .9 \times \times \times$ D-region 2007-10-18 $105.42306 .5 \times \times \times$ 2007-10-18 $115.42306 .9 \times \times \times$ 2007-10-19 09 5.4230 4.7 X X X D-region 2007-10-19 $105.42304 .9 \times \times \times$ 2007-10-19 $115.42304 .7 \times \times X$ 2008-02-05 $\odot 95.42305 .2 \times \times \times$ PMWE 2008-02-05 $105.42305 .3 \times \times X$ 2008-02-05 11 5.4230 NaN X X X 2008-02-06 09 5.4230 4.8 X X X PMWE 2008-02-06 $105.42305 .3 \times \times X$ 2008-02-06 $115.42305 .3 \times x \times$ 
2008-02-07 $115.42304 .9 \times \times \times$

2008-02-08 $115.42304 .9 \times \times \times$ PMWE

2008-02-11 $125.42304 .9 \times$ X X PMWE 2008-02-11 $135.42305 .2 \times \times \times$ 2008-02-12 11 5.4230 4.6 X X X PMWE 2008-02-12 $125.42304 .7 \times \times X$ 2008-03-12 08 5.4230 0.0 X X X PMWE? 2008-03-12 ๑9 5.4230 $0 . \odot \times X X$ 2008-03-12 105.4230 0.0 X X X 2008-05-27 09 5.4230 4.7 X X X PMSE 2008-05-27 10 5.4230 4.9 X X X 2008-05-27 115.4230 4.8 X X X 2008-05-27 $125.42304 .6 \times \times \times$ 2008-05-27 14 5.4230 NaN X X X Test 2008-05-27 $157.9530 \mathrm{NaN} \times \mathrm{X} X$ 2008-05-27 16 5.4230 NaN X X X 2008-05-27 175.4230 NaN X X X 2008-05-27 18 5.4230 NaN X X X 2008-05-30 $095.42304 .8 \times X \times$ XMSE 2008-05-30 10 5.4230 4.7 X X X 2008-05-30 $125.42304 .7 \times \times \times$ 2008-05-30 $135.42304 .7 \times \times \times$ 2008-06-16 08 5.4230 NaN X X X PMSE 2008-06-17 07 5.4230 NaN X X X Tune 2008-06-17 08 5.4230 NaN X X X PMSE 2008-06-17 09 5.4230 NaN X X X 2008-06-17 10 5.4230 NaN X X X 2008-06-17 115.4230 NaN $X X X$ 2008-06-18 07 5.4230 NaN X X X Tune 2008-06-19 10 5.4230 4.8 X X X PMSE 2008-06-19 $115.42304 .7 \times \mathrm{X} X$ 2008-06-26 08 5.4230 NaN X X X PMSE 2008-06-26 ๑9 5.4230 NaN $X X X$ 2008-06-26 10 5.4230 NaN X X X 2008-06-26 115.4230 NaN X X X 2008-06-28 09 5.4230 NaN X X X XMOD 2008-06-28 $105.4230 \mathrm{NaN} \times \mathrm{X} X$ 2008-06-28 $115.4230 \mathrm{NaN} X X X$ 2008-07-03 116.7700 0.० X X X PMSE 2008-09-29 $125.42304 .8 \times \times \times$ Test 2008-09-29 $135.42304 .9 \times \times X$ 2008-09-29 145.42304.9 X X X

2008-10-27 $135.42304 .9 U-U$

2008-10-31 $115.42305 .3 \mathrm{Q} \times \mathrm{X}$ QL plot unsuitable 2008-10-31 $126.20005 .4 \mathrm{Q} \times \mathrm{X}$ 2008-10-31 $135.42305 .0 \mathrm{Q} \times \mathrm{X}$ 2008-10-31 $14 \quad 5.42304 .8 \mathrm{Q} \times \mathrm{X}$

2008-11-03 $116.20005 .4 \mathrm{U}$ U U 2008-11-03 $135.42305 .2 \cup U U$

2008-11-04 12 6.2000 6.0 Q X X QL plot unsuitable

2008-11-08 $185.42305 .9 \mathrm{U}$ U U disturbed conditions 2008-11-08 20 5.4230 5.8 U U U

2008-11-08 $215.42305 .6 \cup U$ U

2008-11-08 $225.42305 .8 \mathrm{U}$ U U

2008-11-26 10 5.4230 4.8 X X X D-region

2009-01-19 $125.42304 .9 \times \times \times$ Test

2009-05-15 $175.42304 .9 \times \times \times$ Test 
2009-05-16 $195.42305 .0 \times \times \times$ Test 2009-05-19 $\odot 95.42305 .2 \times \times \times$ Test 2009-05-19 $125.42305 .5 \times \times \times$ Test 2009-07-22 105.4230 0.๑ X X X PMSE 2009-07-22 11 5.4230 NaN X X X 2009-07-23 08 5.4230 $0.0 \times \times \times$ XMSE 2009-07-23 10 5.4230 NaN X X X 2009-07-23 11 5.4230 NaN X X X 2009-07-24 09 5.4230 NaN $X X X$ PMSE 2009-07-24 10 5.4230 NaN X X X 2009-07-24 11 5.4230 NaN X X X 2009-07-27 10 5.4230 NaN X X X 2009-07-27 11 5.4230 NaN X X X 2009-07-28 09 5.4230 NaN $X X X$ PMSE 2009-07-28 $10 \quad 5.4230 \mathrm{NaN} X X X$ 2009-07-28 11 5.4230 NaN X X X 2009-07-29 $\odot 9$ 5.4230 NaN X X X Partial refl. 2009-07-30 $\odot 9$ 5.4230 NaN X X X PMSE 2009-07-30 $105.4230 \mathrm{NaN} X X X$ 2009-07-30 $115.4230 \mathrm{NaN} X X X$ 2009-07-31 07 5.4230 4.7 X X X Tune 2009-07-31 $\odot 9$ 5.4230 NaN $X X X$ PMSE 2009-07-31 $105.4230 \mathrm{NaN} X X X$ 2009-07-31 11 5.4230 NaN $X X X$ 2009-08-01 $\odot 9$ 5.4230 NaN X X X Partial refl. 2009-08-01 $105.4230 \mathrm{NaN} X X X$ 2009-08-09 $095.42304 .7 \times \times \times$ PMSE 2009-08-09 $105.42304 .7 \times \times \times$ 2009-08-09 $115.42304 .8 \times \times X$ 2009-08-11 $125.42304 .6 \times \times \times$ PMSE 2009-08-11 $135.42304 .7 \times \times X$ 2009-08-12 $\odot 95.42304 .9 \times \times \times$ PMSE 2009-08-12 $105.42304 .8 \times \times X$ 2009-08-12 $115.42304 .9 \times \times \times$ 2009-08-12 $12 \quad 5.42304 .9 \times \times \times$ 2009-12-13 $\odot 67.9530$ ๑.๑ X X X D-region

2010-03-02 $125.42306 .2 \mathrm{U}$ U 2010-03-02 $135.42306 .2 \mathrm{U} \mathrm{U}$ 2010-03-02 $14 \quad 5.42305 .2$ U U U

2010-03-12 105.42495 .4 U U 2010-03-12 125.42495 .7 U U -

2010-03-14 095.42496 .0 U U 2010-03-14 $105.42495 .6 \mathrm{U} \mathrm{U}$ 2010-03-14 $115.42495 .5 \mathrm{U}$ U 2010-03-14 $125.42495 .3 \mathrm{U}$ U 2010-03-14 135.42495 .4 U U -

2010-06-01 08 7.9530 $0.0 \times \times \times$ X Tune 2010-06-01 09 7.9530 $0.0 \times \times \times X$ PMSE 2010-06-09 08 7.1000 6.8 X X X PMSE 2010-06-09 $107.100010 .6 \times \times X$ 2010-06-18 08 7.1000 8.9 X X X PMSE 2010-09-13 $\odot 95.42305 .9 \times \times \times$ Test

2010-09-13 105.42305 .9 U U - radar scanning 2010-09-13 $115.42306 .0 \mathrm{U}$ U 2010-09-13 $125.42306 .0 \mathrm{U}$ U -

2010-09-14 105.42307 .9 U U - radar scanning 2010-09-14 $115.42307 .1 \mathrm{U}$ U 2010-09-14 125.42306 .2 U U - 


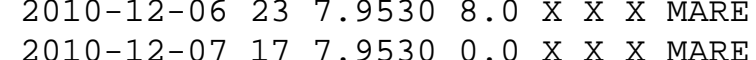

2010-12-07 197.9530 ๑.๑ X X X MARE

2010-12-16 $103.95005 .2 \times \times \times$ Actually A2

2010-12-16 $113.95005 .5 \times \times \times$

2010-12-16 $123.95005 .2 \times \times \times$

2011-03-07 $126.1988 \quad 7.0 \mathrm{Y} Y-$

2011-03-07 13 6.9588 $6.8 \mathrm{U}-\mathrm{U}$

2011-03-07 $14 \quad 6.9588 \quad 7.2$ U U -

2011-05-07 106.20006 .9 U U - radar scanning

2011-05-07 $116.20006 .6 \mathrm{U}$ U -

2011-05-07 126.20006 .5 U U -

2011-05-09 $106.20006 .8 \mathrm{U}$ U - radar scanning

2011-05-09 116.20006 .0 U U -

2011-05-10 $096.20006 .9 \times \times \times$ test

2011-05-10 $106.20007 .5 \mathrm{U} U$ -

2011-05-10 116.20007 .4 U U -

2011-05-10 126.20006 .3 U U -

2011-07-04 11 5.3000 5.8 U U - HF initially $6 \mathrm{~S}$

2011-07-04 $125.30006 .0 \mathrm{U}$ U -

2011-10-05 $125.42307 .3 \mathrm{U}$ U - disturbed conditions 2011-10-05 $135.42300 .0 \mathrm{U}$ U U

2011-10-06 $135.42307 .0 \mathrm{U}$ U 2011-10-06 $145.42305 .8 \mathrm{U}$ U -

2011-10-07 136.19806 .2 U U 2011-10-07 146.19806 .5 Y Y 2011-10-07 $156.7651 \quad 7.0$ Y Y 2011-10-07 $166.77006 .4 \mathrm{Y}-\mathrm{Y}$ 2011-10-07 176.20005 .7 Y Y -

2011-10-08 137.10007 .7 Y Y 2011-10-08 $147.95307 .2 Y-Y$ 2011-10-08 $156.20006 .5 \mathrm{Y}-\mathrm{Y}$ 2011-10-08 $165.42305 .0 \mathrm{U}-\mathrm{U}$

2011-10-09 $126.20006 .2 \times \times \times$ tune

2011-10-09 145.42305 .6 Y Y - radar scanning 2011-10-09 155.42305 .4 Y Y U

2011-10-10 13 6.2000 nan U U - radar scanning 2011-10-10 146.2000 nan $Y U Y$ 2011-10-10 157.1000 nan $Y-Y$ 2011-10-10 $16 \quad 6.2000$ nan $Y-Y$ 2011-10-10 175.4230 nan $Y-Y$ 2011-10-10 18 5.4230 nan $Y-Y$

2011-10-11 $127.10007 .5 \times$ X X CW tune-up

2011-10-11 137.10007 .7 Y Y Y 2011-10-11 $147.95307 .5 \mathrm{Y}-\mathrm{Y}$ 2011-10-11 $157.10007 .2 \mathrm{Y}-\mathrm{Y}$ 2011-10-11 165.42305 .1 U - U 
2011-11-03 127.1001 7.3 Y Y 2011-11-03 136.2001 7.2 $Y Y$ 2011-11-03 146.20016 .1 Y $Y$ -

2011-11-08 107.95309.8 X X X test

2011-11-14 12 6.8800 11.5 X X X test

2011-11-14 13 5.5200 10.3 X X X test

2011-11-15 $10 \quad 7.273011 .7$ Y Y Y 2011-11-15 11 7.2730 11.5 Y Y $Y$ 2011-11-15 127.273011 .4 Y $Y$ Y 2011-11-15 13 7.2730 9.8 Y Y Y

2011-11-16 $116.700010 .3 \mathrm{Y} Y$ 2011-11-16 126.7000 10.3 Y Y 2011-11-16 13 6.7000 8.6 Y Y -

2011-11-17 137.95308 .6 Y $Y$ 2011-11-17 146.2000 7.8 Y Y 2011-11-17 15 6.2000 6.3 Y Y -

2011-11-18 10 7.2730 10.3 Q Q Q 2011-11-18 11 7.2730 11.5 Q Q Q 2011-11-18 126.7000 9.1 Y Y 2011-11-18 13 5.3095 8.5 Y Y 2011-11-18 $146.2000 \quad 6.9$ Y $Y$ 2011-11-18 $156.2000 \quad 5.7$ Y Y 2011-11-19 126.05008 .5 Y Y 2011-11-19 13 6.0500 8.7 Y Y 2011-11-19 14 6.2000 6.5 Y Y -

2011-11-20117.52009.0 X X X tune 2011-11-20 $127.37008 .6 \mathrm{Y} Y$ 2011-11-20 $136.0500 \quad 7.8$ Y Y 2011-11-20 146.20006 .5 Y $Y$ 2011-11-20 $155.52006 .0 \mathrm{Y}$ 2011-11-22 116.700010 .9 Y Y 2011-11-22 126.050010 .5 Y Y 2011-11-22 136.77007 .5 U U -

2011-11-23 126.7000 9.0 Y Y 2011-11-23 136.7000 8.4 Y Y 2011-11-23 14 5.4230 6.5 Y Y 2011-11-23 153.95004 .4 Y $Y$ -

2011-11-24 $105.3095 \quad 8.7$ Y Y 2011-11-24 $116.70008 .6 \mathrm{Y} Y$ 2011-11-24 126.2000 8.3 Y Y Y 2011-11-24 135.52005.1 U U U

2011-11-25 $096.77006 .7 \times \times \times$ tune 2011-11-25 105.42307 .9 U U 2011-11-25 $117.10009 .1 \mathrm{U} U$ 2011-11-25 126.70007 .8 Y Y 2011-11-25 135.30956 .4 Y Y - 

2011-11-26 13 5.3095 7.3 Y Y -

2011-11-27 126.7000 ๑.๑ Y Y 2011-11-27 13 5.3095 0.0 Y Y 2011-11-27 146.20000 .0 U U -

2011-12-07 $095.42307 .1 \times \times \times$ API

2011-12-07 10 5.4230 7.5 X X X 2011-12-07 11 5.42307.3 X X X 2011-12-07 12 5.4230 7.2 X X X 2011-12-07 13 5.42306.9 X X X 2011-12-07 14 5.4230 4.7 X X X 2011-12-08 $\odot 8$ 5.4230 5.0 X X X API 2011-12-08 $095.42306 .4 \times \times X$ 2011-12-08 $105.42307 .7 \times \times \times$ 2011-12-09 08 5.4230 5.0 X X X API 2011-12-09 $095.42306 .3 \times \times X$ 2011-12-09 $105.42307 .3 \times \times x$ 2011-12-09 $115.42307 .2 \times \times x$ 2011-12-09 $125.42306 .4 \times \times x$ 2011-12-09 $135.42305 .1 \times \times x$ 2011-12-11 13 5.4230 5.8 X X X 2011-12-11 $145.42304 .8 \times \times X$ 2011-12-12 08 5.4230 5.4 X X X API 2011-12-12 $095.42306 .1 \times \times X$ 2011-12-12 $105.42307 .1 \times \times x$ 2011-12-12 $115.42307 .9 \times \times \times$ 2011-12-12 $125.42307 .1 \times \times x$ 2011-12-12 13 5.4230 6.4 X X X 2011-12-13 $095.42306 .1 \times \times x$ 2011-12-13 10 5.4230 6.9 X X X 2011-12-13 $115.42307 .1 \times \times \times$ 2011-12-13 $125.42307 .3 \times \times x$ 2011-12-13 13 5.4230 6.4 X X X 2011-12-13 $145.42305 .1 \times \times X$ 2011-12-14 $\odot 95.36005 .8 \times \times X$ API 2011-12-14 $105.36006 .7 \times \times X$ 2011-12-14 $115.36006 .9 \times \times x$ 2011-12-14 $125.36006 .2 \times \times \times$ 2011-12-14 $135.36006 .1 \times \times \times$ 2011-12-15 09 5.3600 6.3 X X X API 2011-12-15 10 5.3600 7.3 X X X 2011-12-15 $115.36007 .1 \times \times \times$ 2011-12-15 $125.36006 .3 \times \times \times$ 2011-12-15 $135.36006 .1 \times \times X$ 2012-01-25 $\odot 9$ 5.4230 NaN X X X PMWE 2012-01-25 10 5.4230 NaN X X X 2012-01-25 $115.4230 \mathrm{NaN} X X X$ 2012-01-25 $125.4230 \mathrm{NaN} X X X$ 2012-01-25 13 7.9530 NaN X X X 2012-01-25 $147.9530 \mathrm{NaN} X X X$ 2012-01-26 10 7.9530 NaN X X X PMWE 2012-01-26 $117.1000 \mathrm{NaN} X X X$ 2012-01-26 $127.1000 \mathrm{NaN} X X X$ 2012-01-26 $137.1000 \mathrm{NaN} X X X$ 2012-01-30 11 5.4230 NaN X X X PMWE 2012-01-30 $125.4230 \mathrm{NaN} X X X$ 2012-01-30 135.4230 NaN X X X

2012-02-12 $136.20007 .1 \mathrm{U}$ U - radar scanning 2012-02-12 $14 \quad 6.20006 .2$ U U - 
2012-02-16 $12 \quad 6.7700 \quad 7.2 \quad \mathrm{Y}$ U $\mathrm{Y}$ 2012-02-16 $13 \quad 6.77006 .9 Y-Y$ 2012-02-16 $14 \quad 6.20005 .8 Y-Y$ 2012-02-16 $15 \quad 5.4230 \quad 5.7 \quad Y \quad U$ Y 2012-02-16 $16 \quad 5.42305 .0 Y-Y$ 2012-02-16 $17 \quad 5.42304 .8 Y-Y$

2012-02-17 $127.10007 .1 \mathrm{Y}-\mathrm{Y}$ 2012-02-17 $13 \quad 6.7700 \quad 7.0 \mathrm{Y}-\mathrm{Y}$ 2012-02-17 $14 \quad 6.2000 \quad 6.6 \quad Y$ Y $Y$ 2012-02-17 $15 \quad 5.4230 \quad 5.7 \quad Y-Y$ 2012-02-17 $16 \quad 5.42305 .2 Y-Y$ 2012-02-17 $175.42305 .6 \mathrm{Y}-\mathrm{Y}$

2012-02-18 $13 \quad 7.1000 \quad 7.3 \quad Y$ U Y 2012-02-18 $14 \quad 6.20006 .5 Y-Y$ 2012-02-18 $15 \quad 6.20006 .6 \mathrm{Y}-\mathrm{Y}$ 2012-02-18 $16 \quad 5.42305 .5 \mathrm{Y}-\mathrm{Y}$ 2012-02-18 $185.42304 .6 \times \times \times$ no UHF radar

2012-02-19 $13 \quad 6.20006 .8 \mathrm{U} U \mathrm{U}$ 2012-02-19 $145.42306 .1 \mathrm{U} U \mathrm{U}$ 2012-02-19 $15 \quad 5.42305 .8 \mathrm{U}-\mathrm{U}$

2012-02-20 $135.42305 .7 \mathrm{U}$ - U disturbed conditions 2012-02-2014 5.4230 $5.1 \mathrm{U}-\mathrm{U}$

2012-02-21 $13 \quad 5.42306 .9 \quad \mathrm{Y} U \mathrm{Y}$ 2012-02-21 $146.20006 .6 Y-Y$ 2012-02-21 $155.42305 .7 \mathrm{Y}-\mathrm{Y}$ 2012-02-21 $165.42304 .9 Y-Y$

2012-02-22 $124.04007 .7 \mathrm{Y} \mathrm{Y} \mathrm{-} \mathrm{radar} \mathrm{and} \mathrm{HF} \mathrm{scanning}$ 2012-02-22 136.77006 .7 Q Q Q no plot?

2012-02-22 $14 \quad 6.20006 .4$ Q Q Q 2012-02-22 $155.30006 .1 \mathrm{Y} Y-$ 2012-02-22 $16 \quad 5.30005 .8$ U U -

2012-02-23 $13 \quad 6.70007 .9 \mathrm{Y} Y$ 2012-02-23 $14 \quad 6.70006 .6 Y Y-$ 2012-02-23 155.30005 .9 Y Y Y 2012-02-23 $16 \quad 5.30005 .9$ U U 2012-02-23 175.30005 .7 U U -

2012-02-24 $13 \quad 6.96007 .9$ Y Y 2012-02-24 $146.20007 .8 Y-Y$ 2012-02-24 $15 \quad 6.20006 .4 \quad \mathrm{Y} U \mathrm{Y}$

2012-02-25 $127.10009 .2 \times \times \times$ tune

2012-02-25 $13 \quad 5.42307 .3 \mathrm{Y} Y$ 2012-02-25 $14 \quad 5.42306 .1 \mathrm{U} U \mathrm{U}$ 2012-02-25 15 5.4230 7.1 Y Y U 2012-02-25 $16 \quad 5.42306 .2 Y-Y$

2012-02-26 $136.77007 .7 \mathrm{Y} \mathrm{Y} \mathrm{-} \mathrm{radar} \mathrm{and} \mathrm{HF} \mathrm{scanning}$ 2012-02-26 $14 \quad 6.7700 \quad 7.3 \quad Y$ Y $Y$ 2012-02-26 $157.10006 .6 Y-Y$ 2012-02-26 $167.10006 .6 \mathrm{Y}-\mathrm{Y}$ 2012-02-26 $17 \quad 5.42305 .8 \mathrm{Y}-\mathrm{Y}$ 
$2012-02-27 \quad 136.20006 .6 \mathrm{U} U \mathrm{U}$

2012-02-28 08 7.1000 $0 . \odot \times \times \times$ X PMWE?

2012-02-28 $117.10006 .3 \times \times \times$

2012-05-21 07 7.1000 7.4 X X X PMSE

2012-05-21 08 7.1000 7.7 X X X

2012-05-21 $097.10007 .5 \times \times x$

2012-05-21 $107.10007 .6 \times \times x$

2012-05-21 $117.10007 .3 \times \times \times$

2012-06-28 $085.42305 .9 \times \times \times$ test 2012-06-28 $096.20006 .0 \times \times \times$ test 2012-06-28 $107.10006 .5 \times \times \times$ test

2012-07-02 08 5.4230 14.7 X X X API

2012-07-02 09 5.4230 5.4 XXX 2012-07-02 $105.42305 .7 \times \times \times$ PMSE 2012-07-02 $115.42305 .7 \times \times X$ 2012-07-02 $125.42305 .5 \times \times X$ 2012-07-03 08 5.4230 5.6 X X X PMSE 2012-07-03 $\odot 95.42305 .7 \times \times X$ 2012-07-03 $105.42305 .6 \times \times x$ 2012-07-03 $125.42305 .9 \times \times \times$ 2012-07-03 13 5.4230 5.4 XXX 2012-07-03 $145.42005 .5 \times \times x$

2012-07-03 $15 \quad 5.30005 .6 \mathrm{U}$ U 2012-07-03 165.30005 .7 U U -

2012-07-04 08 5.4230 6.4 X X X API 2012-07-04 $\odot 95.42305 .8 \times \times X$ 2012-07-04 10 5.42306.0 X X X

2012-07-04 $115.42005 .6 \mathrm{U} U$ 2012-07-04 125.42005 .5 U U -

2012-07-06 08 5.4230 6.3 X X X API

2012-07-06 $095.42306 .4 \times \times X$ 2012-07-06 $105.42305 .8 \times \times x$

2012-07-06 $11 \quad 5.4230 \quad 6.3 \mathrm{U}$ U 2012-07-06 $125.42305 .8 \mathrm{U}$ U -

2012-07-07 $175.42305 .8 \times \times \times$ test 2012-07-09 08 5.4230 $0.0 \times \times \times$ X PMSE 2012-07-09 $\odot 95.42305 .5 \times \times X$ 2012-07-09 $105.42305 .4 \times \times \times$ 2012-07-09 $115.42300 .0 \times \times \times$ 2012-07-09 $125.42304 .9 \times \times \times$ 2012-07-09 $135.42305 .5 \times \times \times$

2012-07-09 155.30005 .9 U U - disturbed conditions

2012-07-10 $095.42304 .7 \times \times \times$ PMSE 2012-07-11 08 5.4230 5.8 X X X PMSE 2012-07-11 $\odot 95.42305 .6 \times \times X$ 2012-07-11 $105.42305 .2 \times \times \times$ 2012-07-13 08 6.7700 6.0 X X X PMSE

2012-09-29 14 5.3000 9.3 X X X test 2012-09-29 $156.20008 .7 \times \times X$

2012-10-10115.42305.9 X X X test 
2012-10-12 $126.20006 .7 U-U$ 2012-10-12 $135.42305 .8 \cup$ U U

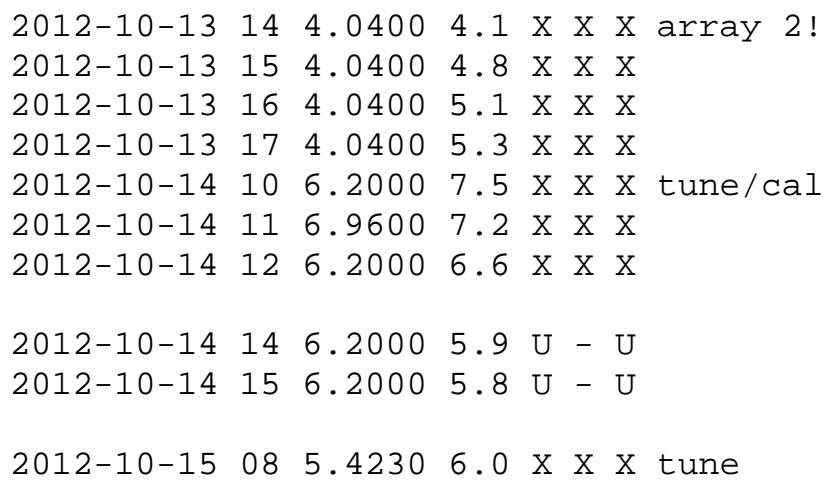

2012-10-21 13 7.9530 9.3 Y U Y radar scanning 2012-10-21 $147.95309 .1 \mathrm{Y} U$ Y radar scanning 2012-10-21 15 7.9530 9.0 Y U Y radar scanning 
2012-10-22 $125.423010 .6 \times \times \times$ tune

2012-10-22 $137.953010 .1 \mathrm{Y} \cup \mathrm{Y}$ radar scanning

2012-10-22 147.95309 .3 Y U Y radar scanning

2012-10-22 $157.95309 .2 \mathrm{Y} \cup \mathrm{Y}$

2012-10-22 $16 \quad 7.10008 .2$ Y Y Y

2012-10-22 $17 \quad 7.10007 .0$ Y Y $Y$

2012-10-24 ๑9 5.42309.6 X X X test

2012-11-23 095.40917 .7 U U -

2012-11-23 106.18618 .5 Y Y -

2012-11-24 $\odot 8$ 5.4091 0.0 U U - disturbed conditions

2012-11-24 09 5.4091 5.8 U U -

2012-11-24 105.40917 .2 U U -

2012-11-24 116.18616 .9 U U -

2012-12-03 $105.60205 .8 \times \times X$ not in handwritten log

2012-12-03 $115.60205 .9 \mathrm{Y}$ - Y large data gap

2012-12-03 $125.60205 .7 \mathrm{Y}-\mathrm{Y}$

2012-12-03 $135.60205 .1 \mathrm{Y}-\mathrm{Y}$

2012-12-04 $115.60205 .7 Y-Y$

2012-12-04 125.60205 .5 Y Y Y

2012-12-04 135.60205 .0 Y Y U

2012-12-05 $115.42305 .2 \times \times x$

2012-12-06 $105.60205 .6 Y-Y$

2012-12-06 115.60205 .5 Y Y Y

2012-12-06 125.60035 .3 Y Y -

2012-12-07 $105.42305 .7 \times \times x$ 2012-12-07 $115.42305 .5 \times \times x$ 2012-12-07 $125.42305 .0 \times \times \times$ 2012-12-07 $13 \quad 5.60564 .9 \mathrm{U}-\mathrm{U}$

2013-02-16 $12 \quad 6.20006 .7 \quad \mathrm{Y} U \mathrm{Y}$ 2013-02-16 $135.30006 .6 \mathrm{Y} \mathrm{U} \mathrm{Y}$ 2013-02-16 $14 \quad 5.30005 .7$ Y Y Y 2013-02-16 $155.30005 .2 Y-Y$

2013-02-17 $125.30005 .6 \cup$ U U 2013-02-17 13 5.3000 5.0 U U U

2013-02-18 $12 \quad 5.30005 .3$ U U 2013-02-18 $135.30005 .5 \mathrm{U} U \mathrm{U}$

2013-02-18 $145.30005 .4 \cup \cup \cup$ but $Y$ after 15:30 UT, A2, X-mode

2013-02-19 126.20006 .9 U U U radar scanning

2013-02-19 $13 \quad 6.20006 .7 \mathrm{U} \mathrm{U} \mathrm{U}$

2013-02-19 $145.42305 .8 \mathrm{U}$ U U disturbed conditions

2013-02-20 $125.35007 .2 \mathrm{U}$ U -

2013-02-2013 5.3500 7.3 U U - disturbed conditions

2013-02-2014 5.4230 5.8 U U U X-mode was A2

2013-02-21 127.10007 .8 Y Y Y 2013-02-21 137.10007 .3 Y $Y Y$ 2013-02-21 145.42306 .8 Y $Y Y$ 2013-02-21 155.42305 .9 Y U Y

2013-02-23 $\odot 8 \quad 5.42305 .8$ U U - 
3-02-23 $09 \quad 6.20007 .0 \mathrm{U} U$ 2013-02-23 $106.2000 \quad 6.6 \mathrm{U}$ U U 2013-02-23 116.20007 .3 Y U Y 2013-02-23 126.20007 .3 Y Y Y 2013-02-23 $13 \quad 5.42306 .6 Y Y-$ 2013-02-23 $14 \quad 6.20006 .9$ Y $Y Y$ 2013-02-23 $155.42305 .7 \mathrm{Y}-\mathrm{Y}$

2013-02-24 115.42305 .9 U U U 2013-02-24 125.42306 .2 U U 2013-02-24 $13 \quad 5.42306 .5 \mathrm{U} \mathrm{U} \mathrm{U}$ 2013-๑2-24 $14 \quad 5.42305 .9 \mathrm{U} \cup \mathrm{U}$

2013-02-25 $08 \quad 5.42306 .5 \mathrm{U} U \mathrm{U}$ 2013-02-25 $\odot 95.42306 .8$ Y U Y 2013-02-25 $106.20007 .3 \mathrm{Y}-\mathrm{Y}$ 2013-02-25 $116.7700 \quad 7.0$ Y Y Y 2013-02-25 $126.7700 \quad 7.2$ Y U Y 2013-02-25 $13 \quad 6.7000 \quad 7.1$ Y $Y Y$ 2013-02-25 $14 \quad 5.4230 \quad 6.3 \quad Y$ Y $Y$ 2013-02-25 155.42306 .0 Y Y $Y$ 2013-02-25 $16 \quad 5.42305 .7 \quad Y$ U Y 2013-02-25 $17 \quad 5.42305 .7 Y-Y$

2013-02-26 08 5.4230 $6.6 \mathrm{U} U$ 2013-02-26 $09 \quad 6.2000 \quad 7.5$ Y Y 2013-02-26 106.20007 .5 Y Y $Y$ 2013-02-26 117.10008 .1 Y $Y Y$ 2013-02-26 127.10007 .7 Y $Y Y$ 2013-02-26 $13 \quad 7.10007 .9$ Y $Y Y$ 2013-02-26 $14 \quad 5.42307 .1$ Y Y $Y$ 2013-02-26 $155.42305 .8 \mathrm{U}$ U U 2013-03-05 126.80007 .9 Y Y 2013-03-05 $136.88007 .8 Y Y$ 2013-03-05 $14 \quad 6.88007 .9$ Y Y 2013-03-05 155.55007 .2 Y Y 2013-03-05 165.55006 .7 U U 2013-03-06 127.95309 .2 U U 2013-03-06 $13 \quad 7.95308 .1 \mathrm{Y} Y-$ 2013-03-06 $14 \quad 6.96007 .7 \mathrm{Y} Y$ 2013-03-06 155.56006 .9 Y Y 2013-03-06 $165.56006 .0 \mathrm{U}$ U -

2013-03-1912 7.95307.9 X X X tune

2013-03-20 $097.95307 .9 \times \times \times$ PMWE 2013-03-20 $107.95307 .5 \times \times \times$ 2013-03-20 $117.95308 .1 \times \times \times$ 2013-03-20 $127.95307 .9 \times \times \times$ 2013-03-21 $\odot 97.95300 .0 \times \times x$ 2013-03-21 $107.95300 .0 \times \times x$

2013-05-15 $086.77006 .4 \times \times \times$ test 2013-05-15 $096.77006 .6 \times \times x$ 2013-05-21 $166.77006 .7 \times \times \times$ test 2013-05-21 $176.77006 .9 \times \times X$ 2013-05-22 $076.77007 .7 \times \times \times$ PMSE 2013-05-22 08 6.7700 $7.6 \times \times \times$ 2013-05-22 $096.77007 .9 \times \times \times$ 2013-05-22 $106.77007 .8 \times \times x$ 2013-05-22 $116.77007 .7 \times \times x$ 2013-05-22 $126.77007 .8 \times \times x$ 2013-05-23 $07 \quad 6.77000 .0 \times \times X$ 2013-05-23 $08 \quad 6.77000 .0 \times X X$ 
2013-06-14 $156.77006 .2 \times \times x$ 2013-06-14 $16 \quad 6.77006 .3 \times \times x$ 2013-06-17 $\odot 96.77007 .6 \times \times \times$ 2013-06-17 $106.7700 \quad 7.1 \times \times \times$ 2013-06-17 $116.77006 .7 \times \times x$ 2013-06-17 $126.77006 .5 \times \times x$ 2013-06-18 $\odot 96.77007 .1 \times \times \times$ 2013-06-18 $106.77007 .0 \times \times x$ 2013-06-18 $116.77006 .6 \times \times \times$ 2013-06-20 $\odot 96.77006 .1 \times \times X$ 2013-06-22 $\odot 96.77006 .0 \times \times X$ 2013-06-22 $106.77006 .0 \times \times x$ 2013-06-23 12 6.77006.1 X X X

2013-07-24 $096.77006 .1 \times \times \times$ 2013-07-24 $116.77006 .1 \times \times X$ 2013-07-25 $106.77006 .5 \times \times x$ 2013-07-25 $11 \quad 6.77006 .0 \times \times X$ 2013-07-25 $13 \quad 6.77006 .2 \times x \times$ 2013-07-27 $126.77006 .1 \times \times x$ 2013-07-29 $096.77006 .5 \times \times X$ 2013-07-29 $106.77006 .6 \times \times x$ 2013-07-29 $116.77006 .2 \times \times \times$ 2013-07-29 $126.77006 .1 \times \times x$ 2013-07-29 13 6.77006.1 X X X

test

PMSE

PMSE

PMSE

PMSE

PMSE

PMSE

PMSE

PMSE

PMSE

2013-10-18 $12 \quad 6.2000 \quad 9.3 \quad Y$ U Y 2013-10-18 $13 \quad 6.2000 \quad 9.1 \quad Y$ U Y

2013-10-19 126.2000 9.2 Y U Y 2013-10-19 13 6.2000 9.3 $Y \cup Y$ also $A 3$ 2013-10-19 $14 \quad 6.2000$ 9.1 $Y U$ Y also $A 3$ 2013-10-19 156.20008 .2 Y $Y$ Y also A3

2013-10-20 126.2000 9.2 Y U Y also A3 2013-10-20 13 6.2000 9.2 Y U Y also A3 2013-10-20 $14 \quad 6.2000$ 9.2 $Y U$ Y also A3 2013-10-20 $156.20007 .7 \mathrm{Y} Y \mathrm{Y}$ also $A 3$ 2013-10-2016 6.2000 6.4 Y Y $Y$

2013-10-21 $107.10009 .5 \times \times \times$ tune

2013-10-21 12 7.9530 9.4 Q Q Q

2013-10-21 13 7.9530 9.3 Q Q Q

2013-10-21 147.9530 9.2 Q Q Q

2013-10-21 157.1000 8.5 Q Q Q

2013-10-22 127.950010 .1 Y Y $Y$ 2013-10-22 13 7.9500 10.3 Y Y $Y$ 2013-10-22 $14 \quad 6.60009 .2 \mathrm{Y} Y$ 2013-10-22 156.70008 .5 Y Y Y 2013-10-22 $167.10008 .2 Y-Y$ 2013-10-22 $17 \quad 7.10007 .0 Y-Y$

2013-10-23 $126.70009 .2 \mathrm{U}$ U - disturbed conditions 2013-10-23 $13 \quad 6.7000 \quad 9.3$ U U 2013-10-23 $145.42309 .0 \cup \cup U$ 2013-10-23 155.42307 .9 Y U Y 2013-10-23 $16 \quad 6.20006 .6$ Q Q Q 2013-10-23 $17 \quad 6.20006 .2$ Q Q Q 

2013-10-24 $16 \quad 6.2000 \quad 7.1$ Q Q Q 2013-10-24 $17 \quad 6.20006 .4$ Y Y Y

2013-10-25 $12 \quad 5.4230 \quad 9.5$ Y Y Y 2013-10-25 $13 \quad 5.4230 \quad 9.8$ Y Y Y 2013-10-25 $14 \quad 6.7000$ 9.2 Y Y 2013-10-25 $15 \quad 6.70008 .9$ Y Y $Y$ 2013-10-25 $16 \quad 6.20007 .6$ Y Y Y 2013-10-25 $17 \quad 6.20006 .2 \mathrm{Y}-\mathrm{Y}$

2013-10-26 $115.423010 .2 \times \times \times$ tune 2013-10-26 $125.42309 .5 \mathrm{Y} Y \mathrm{Y}$ also $A 3$ 2013-10-26 13 5.4230 9.2 $Y Y Y$ also $A 3$ 2013-10-26 $14 \quad 6.70008 .5$ Y $Y$ 2013-10-26 $156.70007 .8 \mathrm{Y}-\mathrm{Y}$ 2013-10-26 $16 \quad 6.9600 \quad 7.6$ Y Y Y 2013-10-26 $17 \quad 6.20005 .9 \mathrm{Y}-\mathrm{Y}$

2013-10-27 $116.770010 .7 \times \times \times$ tune 2013-10-27 13 7.9530 9.2 Y U Y radar scanning 2013-10-27 $147.95009 .0 \mathrm{Y}$ Y - also 15 UT

2013-10-28 $117.953010 .2 \times \times \times$ tune 2013-10-28 13 7.9530 9.3 Y U Y radar scanning 2013-10-28 $14 \quad 7.95008 .5$ Y Y Y 2013-10-28 $156.9600 \quad 7.6$ Y Y Y 2013-10-28 $166.96006 .5 \mathrm{Y}-\mathrm{Y}$ also 17 UT

2013-10-29 $125.42309 .2 \mathrm{Y} Y \mathrm{Y}$ also $\mathrm{A3}$ 2013-10-29 13 5.4230 9.3 $Y$ Y $Y$ also A3 2013-10-29 $14 \quad 7.95008 .9$ Y $Y-$ 2013-10-29 156.20006 .3 Y U Y 2013-10-29 165.42305 .4 U U -

2013-10-30 126.96008 .2 U U 2013-10-30 $13 \quad 5.4230 \quad 5.7$ U U -

2013-10-31 126.96006 .9 Y Y 2013-10-31 $136.20006 .0 \mathrm{U}$ U 2013-10-31 $145.42305 .9 \mathrm{Y} Y-$ 2013-10-31 $155.35005 .4 \mathrm{Y} Y-$ 2013-10-31 165.25005 .1 U U -

2013-11-01 $125.42309 .3 \mathrm{Y} Y-$ 2013-11-01 135.42306 .5 Y Y -

2013-11-02 126.96007 .6 Y Y Y radar scanning 2013-11-02 $136.96007 .0 \mathrm{Y} Y \mathrm{Y}$ 2013-11-02 146.96006 .8 Y $Y Y$ 2013-11-02 156.20006 .1 Y Y $Y$

2013-11-03 $117.95309 .4 \times \times \times$ tune

2013-11-03 127.95308 .9 Y U Y radar scanning; also 13 UT 2013-11-03 $146.9600 \quad 7.8$ Y Y $Y$ 2013-11-03 $156.20006 .5 \mathrm{Y} Y \mathrm{Y}$ 2013-11-03 16 6.2000 6.5 Y Y Y also 17 UT 2013-11-03 18 5.4230 5.0 U U -

2013-11-08 155.25006 .0 U U 2013-11-08 $165.25005 .1 \mathrm{U}$ U U

2013-11-12 $15 \quad 5.25006 .2$ Y Y 2013-11-21 15 5.3500 5.๑ Y Y - 
2013-11-26 $107.95307 .3 \times \times \times$

2013-11-2611 7.95307.3 X X X

2013-12-10 106.20007.8 X X X tune

2013-12-11 $106.96007 .9 \times \times \times$ tune

2013-12-11 $116.96007 .8 \times \times \times$ rapid cycle

2014-02-26 15 7.95309.7 X X X tune 2014-06-26 08 6.7700 7.4 X X X PMSE 2014-06-26 $116.77006 .6 \times \times x$ 2014-06-26 $126.77006 .4 \times \times X$ 2014-06-27 $\odot 96.77006 .1 \times \times \times$ PMSE 2014-06-27 10 6.77006.1 X X X 2014-06-27 $135.30005 .8 \times X X$ 2014-06-30 $\odot 86.77006 .3 \times \times \times$ PMSE 2014-06-30 $096.77006 .2 \times \times X$ 2014-06-30 $106.77006 .3 \times \times x$ 2014-06-3011 6.7700 6.3 X X X 2014-06-30 $126.77006 .1 \times \times X$ 2014-07-01 08 6.7700 6.8 X X X PMSE 2014-07-01 $096.77006 .6 \times \times X$ 2014-07-01 $106.77007 .3 \times \times x$ 2014-07-01 $116.77006 .4 \times \times x$ 2014-07-01 $126.77006 .5 \times \times x$ 2014-07-02 $096.77007 .8 \times \times \times$ PMSE 2014-07-02 $106.77007 .0 \times \times X$ 2014-07-02 $116.77006 .6 \times \times \times$ 2014-07-02 $126.77006 .8 \times \times \times$ 2014-07-03 $116.77006 .4 \times \times \times$ PMSE 2014-07-03 $126.77006 .8 \times \times X$ 2014-07-04 $096.77006 .6 \times \times \times$ PMSE 2014-07-04 $106.77007 .2 \times \times X$ 2014-07-04 $116.77007 .4 \times \times x$ 2014-07-04 $126.77006 .7 \times \times x$ 2014-07-04 $136.77006 .4 \times \times x$ 2014-07-06 $155.42306 .5 \times \times \times$ tune 2014-07-07 08 5.42307.4 X X X tune 2014-07-07 $\odot 9$ 5.4230 7.๑ Q Q Q 2014-07-07 $105.4230 \quad 6.9$ Q Q Q 2014-07-07 $11 \quad 5.42306 .7$ Q Q $Q$ 2014-07-08 07 7.9530 $7.2 \times \times X$ ? 2014-07-08 08 6.7700 7.5 X X X PMSE 2014-07-08 $096.77007 .5 \times \times X$ 2014-07-08 $106.77007 .0 \times \times X$ 2014-07-08 116.20006 .7 Q Q Q 2014-07-08 $12 \quad 6.20006 .7$ Q $Q$ Q 2014-07-09 08 5.3000 7.4 X X X PMSE 2014-07-09 $\odot 96.77007 .0 \times \times X$ 2014-07-09 $106.77006 .8 \times \times \times$ 2014-07-09 $116.77006 .6 \times \times \times$ 2014-07-09 $125.42306 .7 \times \times \times$ 2014-07-11 $076.77006 .3 \times \times \times$ PMSE 2014-07-11 08 6.7700 6.3 X X X 2014-07-11 $096.77006 .5 \times \times \times$ 2014-07-11 10 6.77007.0 X X X

2014-10-14 10 6.20007.2 X X X tune 2014-10-15 ๑9 5.3000 7.0 X X X tune 
2014-10-15 126.20007 .2 U U U

2014-10-16 105.57809 .1 U U 2014-10-16 $115.57809 .0 \mathrm{U}-\mathrm{U}$ 2014-10-16 $125.57809 .3 \mathrm{U}$ U 2014-10-16 13 5.4230 $10.5 \mathrm{U}-\mathrm{U}$ 2014-10-16 147.100010 .7 Y Y Y 2014-10-16 157.1000 9.3 Y Y Y

2014-10-17 $10 \quad 6.960010 .2 \quad \mathrm{U}$ U 2014-10-17 $116.975010 .6 \mathrm{Y}-\mathrm{Y}$ 2014-10-17 $12 \quad 6.9750 \quad 9.8 \mathrm{U}$ U 2014-10-17 $136.9750 \quad 9.2 Y-Y$ 2014-10-17 $14 \quad 7.95309 .2$ Y Y $Y$ 2014-10-17 155.42307 .9 U U U

2014-10-18 $145.42307 .0 \mathrm{U}$ U U 2014-10-19 $\odot 95.57806 .0$ U U 2014-10-19 105.57806 .5 U U 2014-10-20 106.97508 .7 U U 2014-10-20 116.97508 .4 U U 2014-10-20 $126.9750 \quad 7.4$ U U 2014-10-20 $137.10009 .2 \times \times \times$ tune 2014-10-20167.10007.3 X X X tune 2014-10-21 $096.20007 .0 \times \times \times$ tune 2014-10-21 $106.20006 .7 \mathrm{U}$ U U 2014-10-21 116.20007 .4 U U U 2014-10-23 107.1000 9.3 Y Y Y 2014-10-23 117.1000 8.2 Y Y Y 2014-10-23 127.10008 .0 U U U

2014-10-24 $097.95308 .3 \times \times X$ test 2014-10-24 $107.95309 .8 \mathrm{Y} U \mathrm{Y}$ 2014-10-24 117.953010 .1 Y U Y 2014-10-24 $127.95309 .2 \mathrm{U}$ U U

2014-10-25 ๑9 5.42307.7 X X X tune 2014-10-25 $105.57809 .1 \mathrm{U}$ U 2014-10-25 115.57809 .0 U U 2014-10-25 125.57807 .9 U U 2014-10-25 $13 \quad 5.57807 .8$ U U 2014-10-25 145.42307 .9 U U U disturbed conditions 2014-10-25 $155.42307 .6 \mathrm{U} \cup \mathrm{U}$ 2014-10-25 $165.42305 .8 \times \times \times$ tune 2014-10-25 $175.42305 .8 \times \times \times$ 2014-10-26 $08 \quad 6.77007 .9 \mathrm{U} U \mathrm{U}$ 2014-10-26 $097.10008 .5 \mathrm{U} U \mathrm{U}$ 2014-10-26 $106.97509 .4 \mathrm{Y} Y$ 2014-10-26 $116.975010 .3 \mathrm{Y} Y-$ 2014-10-26 126.97509 .5 Y Y 2014-10-26 $13 \quad 6.9750 \quad 8.8$ U U $2014-10-27 \quad 106.975012 .3$ U U 2014-10-27 115.578011 .5 U U - disturbed conditions 2014-10-27 136.975010 .2 Y Y -

2014-11-10 16 6.77006.3 X X X test 2014-11-10 $176.77007 .5 \times \times \times$ test 2014-11-1018 5.42305.0 X X X test

2014-11-11 107.95309.7 X X X test 
2014-11-12 107.953010 .9 U U - radar scanning

2014-11-12 117.953010 .8 U U -

2014-11-12 127.953010 .3 U U -

2014-11-12 $13 \quad 7.10009 .7$ U U -

2014-11-12 147.9530 9.2 Y - Y very brief 0-mode (5 s)

2014-11-12 $156.20008 .9 \mathrm{Y}-\mathrm{Y}$

2014-11-12 17 5.4231 4.8 X X X tune

2014-11-13 127.93919 .7 U U 2014-11-13 13 6.7561 9.0 Y Y 2014-11-13 146.2000 8.3 Y - Y very brief 0-mode (5 s) 2014-11-13 15 5.4230 $6.5 \mathrm{Y}-\mathrm{Y}$ 2014-11-13 $165.42305 .8 \times \times \times$ no radar

2014-11-14 14 5.4230 7.0 Y - Y very brief 0-mode (5 s) 2014-11-14 15 5.4230 5.7 X X X no radar

2014-11-17 08 5.4231 8.3 U U 2014-11-17 09 7.0861 8.5 Y Y 2014-11-17 $107.95309 .5 \times X X$ 2014-11-17 $117.953010 .9 \times \times \times$ 2014-11-17 $127.95309 .0 \times \times \times$

2014-11-18 08 6.7700 7.8 U U 2014-11-18 096.7700 10.2 X X X PMWE

2014-11-18 $107.953010 .2 \times \times \times$

2014-11-18 $117.95309 .9 \times \times \times$ 2014-11-18 $127.95309 .0 \times \times \times$ 2014-11-18 13 5.4230 0.0 X X X 2014-11-19 08 6.1999 7.2 U U 2014-11-19 $\odot 9$ 6.1999 8.5 Y Y 2014-11-19 10 6.2000 9.0 X X X short pulses 2014-11-19 $116.20009 .7 \times \times \times$ 2014-11-1912 6.2000 9.2 X X X

2014-11-20 08 6.7700 7.3 X X X PMWE

2014-11-20 $096.77008 .3 \times \times \times$ 2014-11-20 $117.95309 .6 \times \times \times$ 2014-11-20 $127.95309 .2 \times \times \times$ 2014-11-2013 5.3000 9.3 X X X

2014-11-21 08 6.7700 7.8 X X X PMWE 2014-11-21 $096.77008 .8 \times \times X$ 2014-11-21 107.9530 11.0 X X X 2014-11-21 145.4238 6.1 Q Q Q

2014-11-23 10 6.7700 10.6 X X X PMWE 2014-11-23 $116.770010 .8 \times \times X$

2014-11-24 09 6.7700 9.0 X X X PMWE 2014-11-24 106.7700 10.5 X X X 2014-11-24 $116.200011 .4 \times \times \times$ short pulses 2014-11-24 $126.20009 .4 \times \times \times$ 2014-11-24 $136.20009 .1 \times \times \times$ 2014-11-24 14 5.4230 6.2 U U - disturbed conditions

2014-11-25 $105.30008 .9 \times \times \times$ test 2014-11-25 $116.30009 .2 \mathrm{U}$ U - radar scanning 2014-11-25 $126.30009 .2 \mathrm{U} \mathrm{U} \mathrm{U}$ 2014-11-25 13 6.3000 8.4 U U U 2014-11-25 145.35006 .7 U U - 
2014-11-26 10 6.3000 8.5 X X X short pulses

2014-11-26 $116.30008 .9 \times \times \times$

2014-11-26 $126.30008 .9 \times \times \times$

2014-11-26 13 5.42308.1 X X X

2014-11-27 106.300010 .1 Y Y 2014-11-27 116.300010 .2 Y Y $Y$ 2014-11-27 126.300010 .5 Y Y $Y$ 2014-11-27 13 5.4230 9.1 Y Y Y 2014-11-27 145.42307 .5 Y Y U

2014-11-28 $106.300010 .6 \times \times \times$ test 2014-11-28 11 6.3000 10.5 Q Q Q "lace" program 2014-11-28 $12 \quad 6.3000$ 9.0 Q Q Q 2014-11-28 $13 \quad 6.30007 .6$ Q Q Q

2014-11-29 10 6.2999 10.4 X X X no radar 2014-11-2911 6.2999 10.6 X X X 2014-11-29 $125.30009 .5 \times \times \times$ test?

2014-12-04 09 6.3000 9.0 X X X short pulses 2014-12-04 $107.10009 .0 \times \times \times$ 2014-12-10 $106.30007 .4 \times \times \times$ short pulses 2014-12-10 $116.30008 .4 \times \times \times$ 2014-12-10 $126.30008 .9 \times \times \times$ 2014-12-10 $136.30007 .0 \times \times \times$ 2015-03-10 $157.10009 .0 \mathrm{Y} Y-$ 2015-03-10 $167.10009 .0 \mathrm{Y} Y-$ 2015-03-10 $17 \quad 6.20008 .4 \mathrm{Y} Y-$ 2015-03-10 $18 \quad 5.4230 \quad 6.8$ U U -

2015-03-11 15 5.4230 5.8 U U - disturbed conditions

2015-03-12 $075.42306 .0 \times \times \times$ short pulses

2015-03-12 08 5.42306.7 X X X

2015-03-12 156.20006 .7 U U 2015-03-12 $165.42304 .8 \cup U$ -

2015-03-13 156.20007 .3 U U 2015-03-13 $16 \quad 6.2000 \quad 7.1$ Y Y 2015-03-13 $175.42305 .9 \mathrm{Y} Y-$

2015-03-14 157.10008 .4 Y Y 2015-03-14 $167.10007 .9 \mathrm{Y} Y-$ 2015-03-14 $175.42307 .0 \mathrm{Y} Y-$

2015-04-14 ๑9 6.77008.8 X X X tune 2015-04-14 $107.00008 .4 \mathrm{Y} Y$ 2015-04-14 117.00008 .5 Y Y 2015-04-14 $127.00008 .4 \mathrm{Y} Y-$ 2015-04-14 137.00008 .1 Y Y -

2015-04-15 ๑9 5.42306.7 X X X tune 2015-04-15 $105.60006 .7 \mathrm{U}$ U 2015-04-15 $115.60006 .8 \mathrm{U}$ U 2015-04-16 125.60005 .8 Q Q Q 2015-04-16 $155.55007 .0 \mathrm{U}$ U 2015-04-16 165.55005 .9 U U 2015-04-20 $105.60007 .6 \mathrm{U}$ U 2015-04-20 $115.60007 .5 \mathrm{U}$ U 2015-04-20 $127.00007 .6 \mathrm{Y} \mathrm{Y} \mathrm{-}$ 2015-04-2013 7.0000 7.2 Y Y - 
2015-04-22 107.00008 .2 U U $2015-04-22$ 2015-04-22 135.60008 .5 Y Y -

2015-04-22 $145.60008 .2 \times \times \times$ expt stopped at 14 UT.

2015-04-23 $105.60008 .2 \mathrm{U}$ U 2015-04-23 $115.60008 .6 \mathrm{U} \mathrm{U}$ 2015-04-23 127.00008 .5 U U 2015-04-23 137.00008 .5 U U -

2015-04-24 105.60008 .2 Y Y 2015-04-24 $117.00008 .1 \mathrm{Y} Y-$ 2015-04-24 127.00007 .6 Y Y -

2015-04-27 117.00009 .2 Y Y 2015-04-27 127.00009 .5 Y Y 2015-04-27 137.00008 .8 U U -

2015-07-02 $085.30007 .0 \times \times \times$ tune 2015-07-02 $\odot 96.20007 .4$ U U -

2015-07-03 08 6.7700 7.4 X X X PMSE

2015-07-03 $096.77007 .4 \times \times X$ 2015-07-03 $106.77007 .3 \times \times x$ 2015-07-03 $116.77006 .8 \times \times x$ 2015-07-03 $126.77006 .6 \times \times x$

2015-07-06 $\odot 96.77006 .3 \times \times \times$ PMSE 2015-07-06 $106.77006 .8 \times \times \times$ 2015-07-06 $116.77006 .7 \times \times x$

2015-07-07 $08 \quad 6.77006 .7 \times \times \times$ PMSE 2015-07-07 $\odot 96.77007 .6 \times \times X$ 2015-07-07 $10 \quad 5.4230 \quad 6.7$ U U 2015-07-07 12 6.77006.8 X X X PMSE

2015-07-08 $07 \quad 6.77007 .4 \times \times \times$ PMSE 2015-07-08 08 6.2000 7.4 U U 2015-07-08 $\odot 96.77007 .5 \times \times \times$ X PMSE 2015-07-08 $106.77007 .0 \times \times \times$ 2015-07-08 $116.77007 .0 \times \times x$

2015-07-09 $07 \quad 6.77007 .4 \times \times \times$ PMSE 2015-07-09 08 6.7700 $6.9 \times \times X$ 2015-07-09 $096.77006 .9 \times \times \times$ 2015-07-09 $106.77006 .7 \times \times \times$ 2015-07-09 11 6.77006.4 X X X

2015-07-10 08 6.7700 7.1 X X X PMSE 2015-07-10 $096.77007 .1 \times \times \times$ 2015-07-1012 6.7700 6.3 X X X

2015-07-13 10 6.77006.7 X X X PMSE 2015-07-13 $116.77008 .2 \times \times X$

2015-09-23 $185.423010 .0 \times \times \times$ tune 2015-09-23 $196.77008 .5 \times \times X$ 2015-09-24 $095.42305 .5 \mathrm{U}$ U 2015-09-24 $115.42305 .7 \mathrm{U}$ U U 2015-09-24 $125.42305 .5 \cup \cup U$ 2015-09-25 125.42306 .7 U U - 
2015-10-21 $\odot 96.77008 .2 \times X \times$ tune

2015-10-21 $107.00009 .1 \mathrm{Y} Y$ 2015-10-21 $11 \quad 7.00009 .1 \mathrm{Y} Y$ 2015-10-21 127.00008 .3 Y Y -

2015-10-23 107.00008 .3 Y Y 2015-10-23 $117.00008 .5 \mathrm{Y} \mathrm{Y} \mathrm{-}$ 2015-10-23 127.00009 .2 Y Y -

2015-10-24 $107.00009 .0 \mathrm{Y} Y-$ 2015-10-24 $117.00009 .0 \mathrm{Y} Y$ 2015-10-24 127.00008 .8 Y Y 2015-10-24 137.00008 .7 Y Y -

2015-10-25 $107.000010 .1 \mathrm{Y} Y-$ 2015-10-25 $117.00009 .9 \mathrm{Y} Y-$ 2015-10-25 $127.00009 .4 \mathrm{Y} Y$ 2015-10-25 $13 \quad 7.00008 .9$ Y Y -

2015-10-26 ๑9 5.42308.9 X X X tune

2015-10-26 $105.60009 .4 \mathrm{Y} Y$ - (but not strong) 2015-10-26 $115.60009 .0 \mathrm{Y} Y-$ 2015-10-26 125.60009 .2 Y Y 2015-10-26 $13 \quad 5.60008 .3$ Y Y -

2015-10-28 $126.770010 .4 \times \times X$ tune 2015-10-28 $13 \quad 7.95309 .2 \mathrm{Y} Y \mathrm{Y}$ 2015-10-28 $14 \quad 6.77009 .0 \mathrm{Y} Y \mathrm{Y}$ 2015-10-28 $15 \quad 5.42307 .1$ Y Y $Y$ 2015-10-28 $165.42306 .0 \mathrm{Y}-\mathrm{Y}$

2015-10-29 $117.100010 .0 \times \times \times$ tune 2015-10-29 $126.200010 .4 \times X X$ 2015-10-29 $137.95309 .0 Y$ U Y 2015-10-29 $145.42307 .6 \mathrm{Y}-\mathrm{Y}$ 2015-10-29 $155.42306 .7 \mathrm{Y}-\mathrm{Y}$ 2015-10-29 $165.42306 .3 Y-Y$

2015-10-30 08 6.2000 7.4 X X X tune 2015-10-30 $\odot 96.20008 .0 \mathrm{Y} Y-$ 2015-10-30 $107.10008 .5 Y-Y$ 2015-10-30 $11 \quad 7.10009 .5$ Y Y Y 2015-10-30 127.95309 .6 Y $Y$ Y

2015-10-31 $117.10009 .3 \times \times \times$ tune 2015-10-31 $125.42308 .5 \times X X$ 2015-10-31 137.10007 .2 Y Y Y 2015-10-31 145.30007 .0 Y Y $Y$

2015-11-02 11 5.42308.2 X X X tune 2015-11-02 $125.42308 .9 \times X X$ 2015-11-02 $13 \quad 6.77007 .8$ Y Y U

2015-11-03 106.77009 .3 Y Y U 2015-11-03 $116.30009 .4 \mathrm{U}-\mathrm{U}$

2015-11-05 $116.77008 .4 \mathrm{U} \cup \mathrm{U}$ disturbed conditions 2015-11-05 $125.42307 .6 \cup U$ -

2015-11-06 $115.30006 .4 \mathrm{U}$ U U 2015-11-06 $125.30006 .4 \mathrm{U} U \mathrm{U}$ 2015-11-06 $135.30005 .6 \cup U$ - 
2015-11-16 $105.4230 \quad 6.4$ U U 2015-11-16 115.42307 .3 U U -

2015-11-18 106.77007 .9 U U 2015-11-18 126.77009 .2 U U 2015-11-18 135.42308 .4 U U -

2015-11-26 116.20007 .0 U U 2015-11-26 125.30006 .7 U U 2015-11-27 116.20007 .0 U U 2015-11-27 125.42306 .6 U U -

2015-12-04 $115.42306 .0 \mathrm{U}$ - U radar scanning 2015-12-04 $125.42305 .8 \mathrm{U}$ U U

2016-03-04 13 6.20007.1 X X X tune

2016-03-04 146.96008 .1 Y U Y

2016-03-04 $156.20007 .8 Y$ Y $Y$

2016-03-04 $16 \quad 6.20006 .0 \mathrm{Y} Y \mathrm{Y}$

2016-03-09 $105.36006 .6 \mathrm{U}$ U 2016-03-09 $115.36007 .1 \mathrm{U}$ U 2016-03-09 125.36007 .0 U U -

2016-03-10 $105.30006 .3 \mathrm{U} U$ 2016-03-10 $115.30006 .2 \mathrm{U}$ U 2016-03-10 $125.30006 .3 \mathrm{U}$ U 2016-03-11 $105.36006 .1 \mathrm{U}$ U 2016-03-11 115.36006 .1 U U 2016-03-11 125.36006 .6 U U -

2016-04-22 $084.04005 .5 \times \times \times$ test 2016-04-22 $125.30005 .7 \times \times x$

2016-09-23105.20006.1 X X X tune

2016-09-24 115.90006 .6 U U 2016-09-24 125.60006 .2 U U 2016-09-24 $135.30005 .8 \mathrm{U}$ U 2016-09-24 145.30005 .6 U U -

2016-10-12 $115.42306 .4 \times \times \times$ tune 2016-10-12 $125.60006 .1 \times \times \times$ short pulses 2016-10-13 $106.96008 .9 \times \times \times$ tune 2016-10-13 $116.77007 .0 \times \times \times$ short pulses 2016-10-20 $115.42306 .1 \times \times \times$ tune 2016-10-20 125.42306 .0 Q Q Q 2016-10-20 13 5.3000 5.7 Q Q Q but WAILES on A2 later

2017-07-10 18 5.4230 4.8 X X X API 2017-07-1019 5.4230 4.7 X X X 2017-07-11 06 5.4230 5.4 X X X API 2017-07-11 09 5.4230 $0.0 \times \times X$

2017-10-18 104.0400 5.9 Q Q Q 2017-10-18 $11 \quad 5.4230 \quad 6.4$ Q Q Q 2017-10-18 $12 \quad 5.42305 .6$ Q Q Q 2017-10-18 13 5.4230 5.2 Q Q Q 2017-10-18 $14 \quad 5.4230 \quad 4.7$ Q Q Q 2017-10-19 105.54006 .8 Q Q Q 


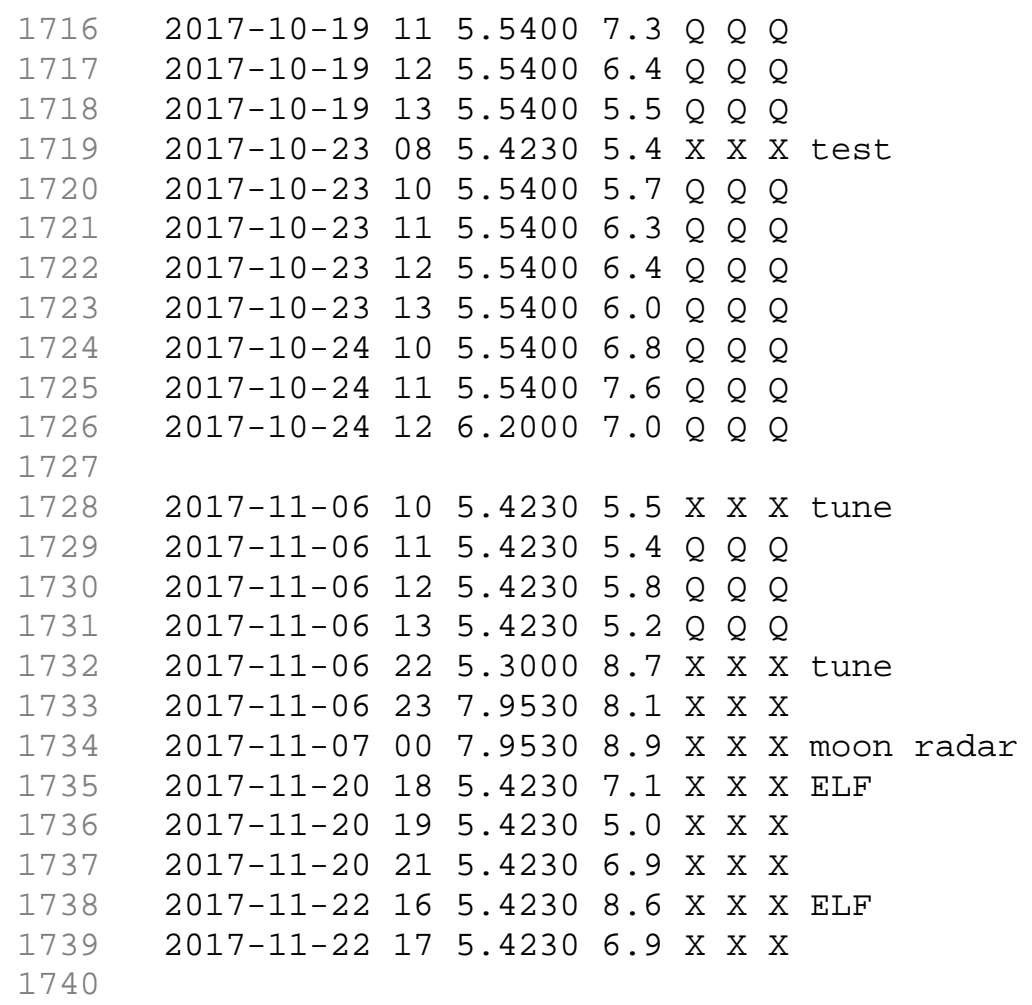

2017-10-19 115.54007 .3 Q Q Q

2017-10-19 125.54006 .4 Q Q Q

2017-10-19 135.54005 .5 Q Q Q

2017-10-23 08 5.4230 5.4 X X X test

2017-10-23 105.54005 .7 Q Q Q

2017-10-23 115.54006 .3 Q Q Q

2017-10-23 125.54006 .4 Q Q Q

2017-10-23 135.54006 .0 Q Q Q

2017-10-24 105.54006 .8 Q Q Q

2017-10-24 115.54007 .6 Q $\quad$ Q

2017-10-24 12 6.2000 7.0 Q Q Q

2017-11-06 $105.42305 .5 \times \times \times$ tune 2017-11-06 115.42305 .4 Q Q Q

2017-11-06 125.42305 .8 Q Q Q

2017-11-06 13 5.4230 5.2 Q Q Q

2017-11-06 22 5.30008.7 X X X tune 2017-11-06 23 7.95308.1 X X X

2017-11-07 $\odot \odot 7.95308 .9 \times \times \times$ moon radar 2017-11-20 18 5.4230 7.1 X X X ELF

2017-11-20 $195.42305 .0 \times \times \times$

2017-11-20 21 5.42306.9 X X X

2017-11-22 16 5.4230 8.6 X X X ELF 1740 